\title{
Active Manipulation of Users in Haptic-Enabled Virtual Environments
}

\author{
Mehrdad H. Zadeh and David Wang \\ Electrical and Computer Engineering \\ Department \\ University of Waterloo \\ \{mehrdad,dwang\}@kingcong.uwaterloo.ca
}

\author{
Eric Kubica \\ Department of Systems Design Engineering \\ University of Waterloo \\ ekubica@kingcong.uwaterloo.ca
}

\begin{abstract}
The main goal of this research is to study the effect of subthreshold forces on human performance in a haptic-enabled virtual reality system. A multi-modal task similar to Fitts is used to study the effects of the sub-threshold forces on user performance. Each user's movement is manipulated using controlled forces such that the user is not aware of the forces. Subjects can see the position of the haptic probe in a virtual environment where they are manipulated using sub-threshold forces. The multi-modal task is used to measure the accuracy of subjects in two experiments. During the experiments, the effects of force intensity and the relative direction of applied forces to the direction of user's hand motion in the presence of visual cues are investigated. A performance index is also introduced that can be used to evaluate human performance in the application of subthreshold forces. A psychophysical method is utilized to ensure that the applied forces on the user's hand are below the force threshold of the human haptic system. Results indicate that user performance is affected by both the intensity and direction of sub-threshold forces even when the users could control their actions through visual feedbacks.
\end{abstract}

\section{Categories and Subject Descriptors}

H.5.2 [User Interfaces]: Haptic I/O; H.1.2 [User/Machine Systems]: Human factors

\section{General Terms}

Human factors, haptic, human performance, sub-threshold force, Perception and psychophysics, human-computer interaction, velocity

\section{INTRODUCTION}

In virtual reality applications, a user usually grasps an input device to explore inside of a virtual environment. A

Permission to make digital or hard copies of all or part of this work for personal or classroom use is granted without fee provided that copies are not made or distributed for profit or commercial advantage and that copies bear this notice and the full citation on the first page. To copy otherwise, to republish, to post on servers or to redistribute to lists, requires prior specific permission and/or a fee.

AMBI-SYS 2008, February 11-13, Quebec, Canada

Copyright @ 2008 ICST 978-963-9799-16-5

DOI 10.4108/ICST.AMBISYS2008.2910 virtual probe or a tool tip usually represents the user's hand in the virtual environment. The user can see the tool tip through a computer or a head-mounted display. Thus, users can only maintain their movement and accuracy using visual cues. However, in a haptic-enabled virtual environment, users can explore and manipulate virtual objects using the sense of touch. Users grasp the end-effector of the haptic device and move it. The haptic device must present appropriate force feedback to the users' hand based on the movement of the users' hand. Haptic-enabled virtual environments are widely used in a variety of applications such as computer-aided design [6], computer-aided assembly [8], computer-assisted surgery [18] and so on. In most of these applications, human performance efficiency in virtual environments is critical to carry out a task [27].

For example, a possible application is tele-surgery where a surgeon wants to carry out a very accurate surgery at a patient site in a remote location. The surgeon and patient are connected through the Internet and the surgeon can see the other site through a display. In this type of application, the surgeon must be able to move freely and interact with the different parts of patient's body. In addition to visual feedback, haptic feedback can also be provided to the surgeons to improve their performance. The sense of touch may be transmitted over the Internet to help a surgeon to better recognize the parts of the patient's body [13]. The surgeon must accurately carry out the task. The main challenge in this type of application occurs when users try to maintain their accuracy in the presence of time-delays over the Internet. Due to the time-delays, it is difficult to carry out tasks efficiently [29].

To develop such applications, an active manipulation of the user (e.g. surgeon) response can be incorporated to maintain the accuracy of the user. In other words, control strategies can be utilized for pulling or pushing the users' hand into predictable responses in order to help the user maintain safe trajectories. However, the feeling of the user must be similar to an actual feeling in a real world situation [23]. The feelings would be unreal if the users feel that the haptic device is pushing or pulling them during a task. Therefore, the goal is to find an approach that the user is manipulated without being aware of it. To achieve this goal, sub-threshold forces can be incorporated in the controlling of users. The sub-thresholds (subliminal) forces are forces that cannot be perceived by the user [24]. In other words, they are below the force threshold of the human haptic system. Sub-threshold forces would be useful in virtual reality applications because the users cannot feel the application 
of these forces on their hands. Lawrence et. al [15] used sub-threshold forces in a friction compensation technique in the design of haptic devices. If one chooses controller gains so that the friction is under-compensated, then the user will still feel the residual effects. However, if the the friction is over-compensated for, then the user will feel an unnatural pulling. Thus, there is a range of friction compensation values that will allow the control strategy to feel transparent to the user.

On the other hand, both force intensity and the relative direction of force to the direction of user's hand motion must be taken into account to convey the properties of virtual environments [10]. Many studies have investigated the human force perception with respect to the intensity, not the direction, of forces $[12,19,20]$. However, the force directions are important in the haptic rendering of virtual objects. It is more critical in haptic display of surface friction, and viscosity of virtual environments that force direction depends on the movement direction and velocity [26]. The relevant works are reviewed in detail in Section 2.

As mentioned, the main goal of this research is to study the effects of intensity and direction of applied forces on human performance by utilizing knowledge of the human haptic perception process. To achieve this goal, a method of psychophysics must be utilized to ensure that the applied forces are sub-threshold forces. The differential force threshold or Just Noticeable Difference (JND) is a measure of the minimum difference between two stimuli that is necessary in order for the difference to be reliably perceived by a user. The first stimulus is called the base stimulus, and the second stimulus is an increment/decrement of the base stimuli. In this study, the focus is on force as a stimulus. The first stimulus (force) called base force, and the second stimulus is an increment/decrement of the base force [9]. The experiments, results, and possible sources of errors are presented and discussed in Section 4 and Section 5, respectively.

In this paper, a multi-modal task is defined for a user interacting with a virtual environment. The user engaged in a task that is similar to the original Fitts task [7] in a virtual environment. The original Fitts task is introduced and explained in Section 3. The multi-modal task is used to evaluate user performance in the presence of the forces that are below the force JND of the human haptic system. Subjects can see the position of haptic probe in the virtual environment when they are manipulated using sub-threshold forces. Essentially, we investigate the effects of unnoticed haptic effects in the presence of visual cues. A performance index is determined that can be used to evaluate human performance in the application of sub-threshold forces. The implications of findings, conclusions and future studies are presented in Section 6.

\section{RELATED WORKS}

As mentioned in the previous section, haptic is used in many applications to improve human performance in virtual reality systems. Salb et al.[22] studied the haptic mediation of the risk values which has been realized using a haptic device. Interventions always cause risks to the patients during a surgery. Critical steps within an intervention can be estimated using preoperative simulations. The risk potentials could be explored with the haptic device to improve an overview over the risks when planning surgical interventions for the target area.

Wall et al. [28] studied the quantitative effects of haptic on user performance. They used Fitts' law in conjunction with another task to develop a measure of human performance in a target task. They found that applied forces can improve the movement times for a difficult task, but had no effect on the index of performance (IP) as defined by Fitts' law. However, they also found a significant improvement in IP by adding haptic effects to a less difficult task. However, we study the sub-threshold haptic effects on human accuracy in a Fitts task by measuring the number of human errors , instead of IP, during the task.

Lee et. al [17] studied the weakest effects that can provide meaningful information to the user. They utilized an adaptive threshold algorithm to determine the lowest detectable forces rendered in the horizontal plane by a low-power, lowfriction, and high-precision haptic device. They investigated the effect of small forces on human performance that can be useful for adding haptic interfaces to smaller systems, e.g. laptops, PDA's, and even cell phones. They used Fitts' Law to analyze performance. In our paper, the effect of small (sub-threshold) forces is investigated using Fitts task. However, the focus is on user performance when the user does not perceive the small forces.

Zadeh et. al [31] determined the absolute force thresholds of the human haptic system for application to a psychophysically motivated lossy haptic (force) compression technique. The compression technique store the haptic data only when the force exceeds a certain threshold of the human haptic perception. They found that, when a user's hand is in motion, fewer haptic details are required to be stored, calculated or transmitted. However, in this paper, the focus is on the effects of forces less than the differential force threshold (JND).

Many researchers have found the JNDs for forces using different kinds of force-feedback devices. Jones [12], in a force matching experiment about the elbow, found a JND ranging between $5 \%$ and $9 \%$ over a range of different base force values. Pang et al. [19] found a JND that lies between 5\% and $10 \%$ for pinching motions between the finger and thumb with a constant resisting force over a range of different base force values between 2.5 and $10 \mathrm{~N}$. Allin et al. [4] used the PHANToM $^{T M}$ haptic device to measure a JND force in a virtual environment. Their goal is to use the thresholds of force sensitivity to construct therapeutic force feedback distortions that stay below these thresholds. They conclude that the visual feedback distortions in a virtual environment can be created to encourage increased force productions by up to $10 \%$, without a patient's awareness.

Most of the work reviewed study the haptic display of stationary rigid objects interacting with the operator's hand. However, motion is important in many virtual reality applications. The dependence on velocity is observed when a user is sliding on the surface of a virtual object or is in contact with a moving object. Another application is the haptic rendering of deformable objects. In this type of application, a user can penetrate into the object. Forces are applied both in and against the direction of the user's motion in a deformable object. In all these applications, unlike previous research, the motion of a user's hand and the virtual haptic object is important. The result in $[16,11,31$, 30] shows a significant variation in human perception and 
the force thresholds while the velocity of the subject's hand is changed, albeit in a different scenario than is studied in this paper.

Another example is the haptic rendering of sliding friction in a virtual reality application. Williams[21] developed several activities with haptic feedback to augment the teaching of standard high school physics concepts. One activity allowed the user to feel different levels of sliding friction such as coarse, medium, fine, and ice. One method to haptic display of sliding on ice is to apply a certain amount of force to the user's hand in the direction of user's hand movement. However, for the coarse level, a certain amount of force is applied to the user's hand in opposed to the direction of user's movement. Thus, the relative direction of applied force to the direction of user's hand motion is critical in the haptic applications. Zadeh et. al [30] derived Just Noticeable Differences (JND) for human force sensing. They found that the effect of force direction on the force JND is dependent on the increasing or decreasing of the force.

\section{FITTS' LAW}

Fitts [7] conducted a systematic analysis on the relationship between accuracy and speed. In the original Fitts task, subjects were asked to tap a pencil-shaped object alternately between two rectangular plates by moving their hand left and right as rapidly as possible for a predetermined duration. The width of the targets $(W)$ and the amplitude of the movement between the targets $(A)$ could be changed by the experimenter to generate a large number of combinations of A and W. Each time a subject tapped one of the targets, a hit is scored. Subjects were asked to score as many hits they could. On the other hand, an additional error plate was mounted on either side of each target plate in the original Fitts task. If subjects hit either of the side plates an error was recorded. An Undershoot or overshoot error occurred, if they hit the side plate that was mounted before or after the target plate, respectively. Subjects were asked to accurately carry out the task to limit misses to no more than about $5 \%$. In other words, they must emphasize accuracy rather than speed.

Fitts presented a model of human motor behaviour. His model is a descriptive model that provides a descriptive measure of human motor performance or throughput (Index of Performance: $I P)$. His model is also a predictive model that provides a prediction equation for the time to acquire a target, given the distance and size of the target. Fitts suggested Equation 1 as the relationship between the target width $(W)$, the amplitude $(A)$ of the movement, and the resulting average movement time $(M T)$ [25].

$$
M T=a+b\left[\log _{2}\left(\frac{2 A}{W}\right)\right]
$$

MT is calculated as the trial duration divided by the number of taps in that time $(s / \operatorname{tap}) . a$ and $b$ are the empirical constants of a linear equation. $I D=\log _{2}\left(\frac{2 A}{W}\right)$ called the index of difficulty $(I D)$. ID represents the difficulty of the task. The $I D$ of a task is related to the distance (Amplitude of Movement) and the width of the target. The larger the A, the harder the task; The larger the $W$, the easier the task. Fitts conducted a series of experiments with different $I D$ s (from 1 to 7 ). The Index of Performance $(I P)$ or

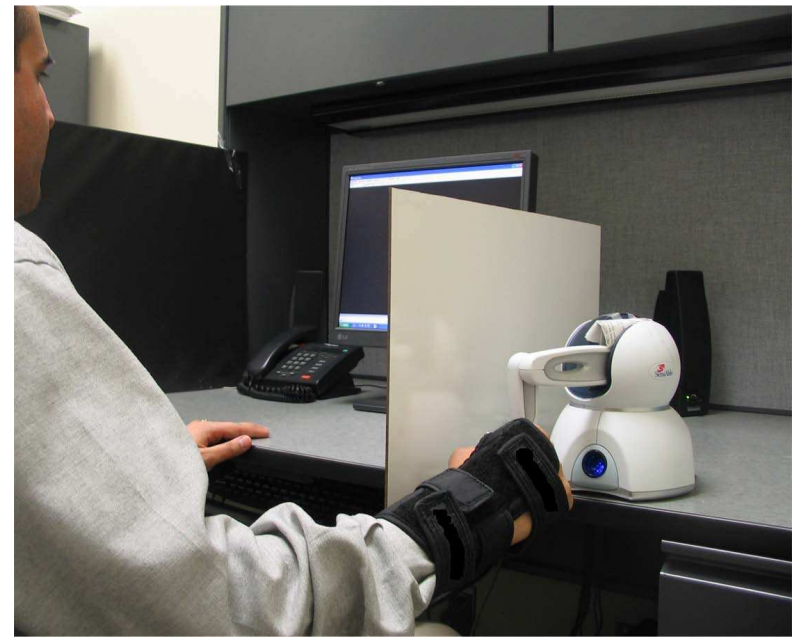

Figure 1: The experimental setup: the PHANToM ${ }^{T M}$ haptic device and a subject.

throughput is presented in Equation 2. Fitts suggested that, for a particular test condition, throughput should be relatively constant over a wide range of task difficulties. In other words, the $I D-M T$ relationship is linear. In addition to $I P$, the accuracy of the user can be evaluated by incorporating the number of hits, overshoots, and undershoots.

$$
I P=\frac{I D}{M T}
$$

As mentioned, the focus of our study is on applications where the user and the object are in relative motion. It is difficult to generate precise mathematical description of behaviour in human motor control applications. Therefore, a reliable motor behaviour task (law) is required to study human motor control behaviour. Fitts is selected to be used in this study because it represents a fundamental relationship that governs many kinds of motor behaviour [25].

\section{METHODS}

\subsection{Participants}

There were 6 participants between the ages of 25 and 39 . All were regular computer users and students at the University of Waterloo. They were right-handed, and had no more than trivial previous exposures to haptic interfaces. The participants did not have any neurological illness or physical injury that would impair hand function or force control ability. The participants were recruited by word of mouth and received $\$ 10$ for their participation in each session. The experiment was conducted in accordance with the University of Waterloo ethical guidelines.

\subsection{Apparatus and Material}

In the experiments, PHANToM ${ }^{T M}$ Omni device [2] was used as the force-feedback device. Softwares were run on 


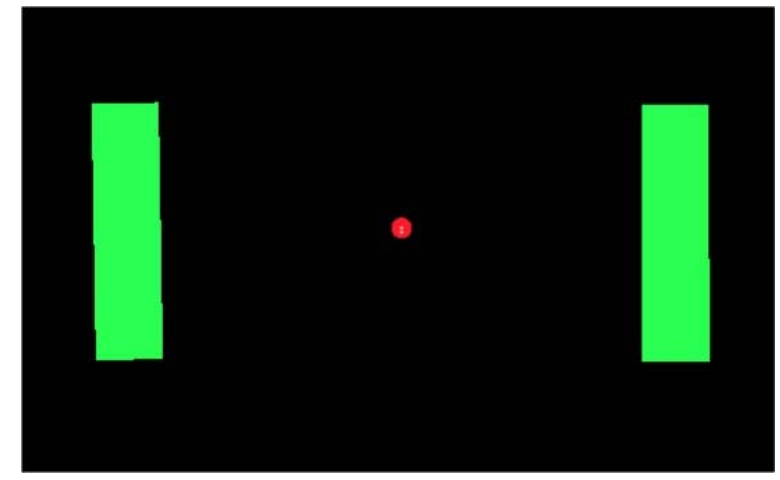

Figure 2: The experimental setup: the 2D virtual environment.

two $3 \mathrm{GHz}$ Pentium 4 computers running Windows XP, and were developed in MATLAB using the Prosense toolbox [1]. Each subject was seated on a chair facing a computer display and asked to place their right elbow on a side support. The wrist of the right hand was restrained with a wrist guard, as shown in Figure 1, so that wrist movements were locked (to make sure that each subject rotated their hand about their elbow). The subject grasped the end-effector of a haptic device. Once each subject was seated comfortably, their right arm and fingers were shielded from their own view with an opaque barrier.

Attention was directed to a 17 " LCD monitor placed approximately $70 \mathrm{~cm}$ from the subject. The monitor contained a graphical display, as shown in Figure 2. The display showed a 2D virtual environment that was used in this experiment. Subject engaged in a task that is similar to Fitts task [7] in a virtual environment. The original Fitts task was done for several indexes of difficulty. However, a fixed index of difficulty, 4, was chosen in this study because we investigated the sub-threshold force effects on the accuracy of subjects in a task with the same difficulty in all experiments. The ID was selected based on the work space of the haptic device. As shown in Figure 3, the 2D virtual environment contained a red ball and two green rectangles. The center-to-center distance between the targets was 10.2 $\mathrm{cm}$, and the width of the target was $1.3 \mathrm{~cm}$. The 2D ball moved when the subject moved the haptic device. During each trial, subjects were asked to tap the two targets (green rectangles) by moving their hand to left and right. Each time the ball was within one of the targets, a hit was scored by subjects. Subjects were asked to score as many as hits they could.

As mentioned in Section 3, in the original Fitts task, subjects were asked to carry out the task as rapidly as possible for a predetermined duration because the objective was to investigate the trade off between velocity and accuracy [7]. In this study, the subjects were also asked to accurately carry out the task in the presence of sub-threshold applied forces. However, unlike Fitts task, subjects were required to maintain their hand's velocity within a specified range to eliminate the effect of velocity on the accuracy of subjects. In other words, the goal was to investigate the effect of sub-threshold forces, not the subject's hand velocity, on the accuracy of subjects. Therefore, the velocity range was set by the experimenter to $0.16-0.20 \mathrm{~m} / \mathrm{s}$ as the reference

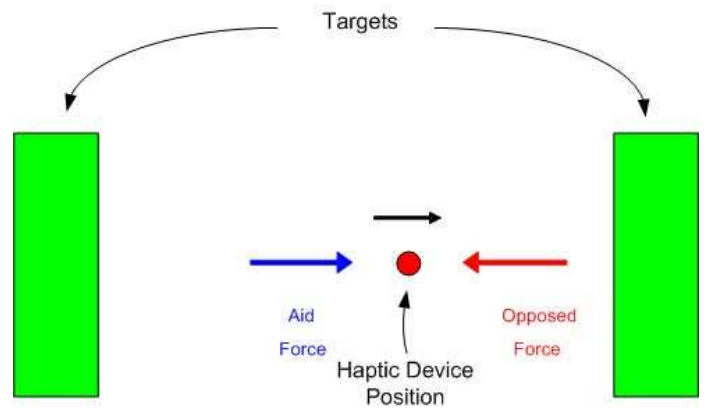

Figure 3: The relative direction of applied forces to the direction of user's hand movement

velocity range.

\subsection{Stimuli}

In this study, the focus is on force as a stimulus. The force stimuli were presented using PHANToM ${ }^{T M}$ haptic device. As mentioned in Section 1, the effects of both force intensity and relative direction of force to the direction of user's hand motion were studied in though two experiments.

The experimenter was able to change the intensity of force through a computer, and apply forces to the subject's hand. As shown in Figure 5, the first force stimulus (base force: F) was a $150 \mathrm{mN}$ force that was continuously applied to the subject's hand from the beginning of each trial. The first interval of measurement period lasted 15 Seconds. Then, the second force stimulus that was an increment/decrement of the base force $(\mathrm{F} \pm \Delta \mathrm{F})$ was applied to the subject's hand in the second interval of measurement period. The second interval also lasted 15 Seconds. For instance, the applied forces on participant ES's hand are presented in Figure 4. The $\Delta \mathrm{F}$ of first trial for both increment and decrement runs were $20 \mathrm{mN}$ in all experiments. However, the $\Delta \mathrm{F}$ for the rest of trials were determined based on the responses of subjects through the experiments. The applied forces in both periods for participant ES are depicted in Figure 4.

The effect of relative direction of force to the direction of user's hand motion was studied via two experiments. As shown in Figures 3, two forces vectors were used in the two experiments. The first one was called the opposed force that was opposed to the direction of the subject's hand movement. It was continuously applied to the subject's hand during each trial in the first experiment. The other one was called the aid force that was continuously applied to the subject's hand in the same direction as the subject's hand movement during each trial in the second experiment. The detail of experiments is presented in Section 4.5.

\subsection{Design}

The effects of force direction and the increment/decrement of the forces were studied through two experiments. The procedure in 4.5 was repeated two times for two different force directions (opposed and aid). Therefore, each subject was required to participate in two experiments plus a onehour training session. The training session was delivered that helped subjects to get familiar with the task. It was required for subjects to carry out the task with less than 


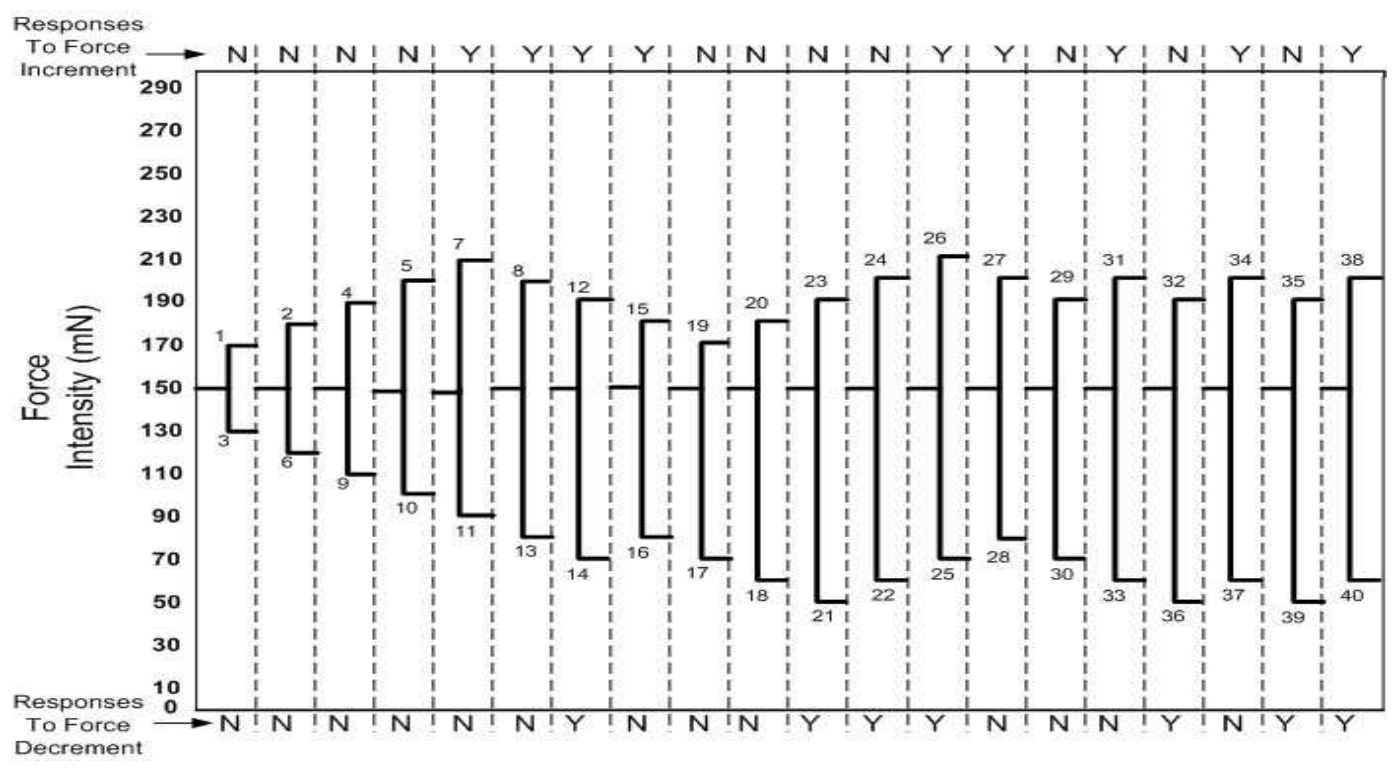

Figure 4: The staircase of forces for participant ES in the aid experiment: the trial sequence numbers of force increment and decrement are shown on the top and bottom of stairs, respectively. The sequence of trials is randomly determined before each experiment. The responses of participant are presented on the top and bottom of figure for force increment and decrement trials, respectively.

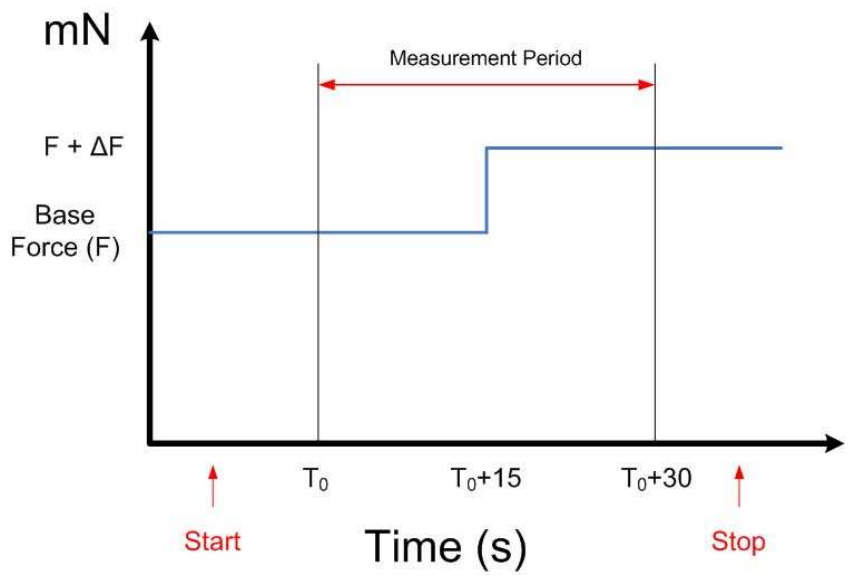

Figure 5: The force stimuli in a force increment trial.

$15 \%$ missing error at the end of training session. A Repeated Measures completely randomized two-factor design was employed in the experiments. All subjects carried out the two experiments in our repeated-measures experiment design. However, the six participants were randomly divided into two three-member groups. The subjects in one group first did the opposed force experiment, then carried out the aid experiment. The subjects in the other group did the aid experiment, then carried out the opposed experiment.

\subsection{Procedure}

In both experiments, the effects of increment or decrement in applied forces on human performance were studied using the Interweaving Staircase (IS) [5] method from psychophysics. Each experiment had two runs (increment and decrement), and was done in 40 trials, 20 trials for each of force increment and decrement runs. The trials of the experiment for one of the subjects are presented in Figure 4. The trials with a force increment and decrement called force increment trials and force decrement trials, respectively. In each trial the subjects were asked to grasp the handle (endeffector) of haptic device and start the task when hear start from the experimenter. They were also asked to stop and leave the handle when they hear stop from the experimenter. A sample trial is presented in Figure 5, and start and stop points are also shown in the figure. The base force was applied to their hand from the beginning of each trial. The measurement period contained two 15-second intervals (before and after increment/decrement of the base force). The first interval of measurement period started when the experimenter ensured that the subjects maintained their velocity within the reference range. The experimenter could monitor the end-effector of haptic device on another computer.

The second interval was started if the missing error of the subject was less than $15 \%$. The missing error equales the sum of overshoots and undershoots divided by the total hits, overshooots, and undershoots that the subject made in the first interval. If the missing error was greater than $15 \%$, the experimenter discarded the data, and started over the first interval. It was also assumed that the subjects maintain their missing error within the 30 -second measurement period. After the first interval, the experimenter randomly incremented/decremented the continuous applied base force according to the IS method. The number of hits, overshoots, undershoots, and the average of subject's hand velocity were separately measured during each interval of measurement period.

In this study, the IS method was utilized to determine the applied forces on the user's hand that were below the force threshold of the human haptic system. As shown in 
Figure 4, the experimenter starts by presenting a sequence of forces (the base force and the increment/decrement force) which progressively increases or decreases in value (steps of $10 \mathrm{mN}$ )based on the response of subject in the previous trial. The sequence of forces were randomly chosen by the experimenter before starting the experiment. For instance, for participant ES, trials 1 and 2 were force increment trials, and trials 3 and 6 are force decrement trials. The subjects were asked to report at the end of each trial if they felt any changes in their feeling. Subjects could respond with Yes, increment, or Yes, decrement or No to detecting changes in force value. The responses of participant ES are presented on the top and bottom of Figure 4 for the increment and decrement trials, respectively. When the subject's response changed from Yes to No, the direction of the force sequence was reversed from ascending to descending, or vice versa. For example, as shown in Figure 4, a $210(150+60) m N$ force was applied on the subject's hand in trial 7 , and the force increasing direction was changed because the subject's response was Yes. In trial 8, the applied force was decreased by $10 \mathrm{mN}$, and a $200 \mathrm{mN}$ force was applied to the subject's hand. In this paper, the trials with a No and Yes responses are called unnoticed and noticed trials, respectively.

The main advantage of the IS method is to reduce the possible biases compared to the original staircase method. In the IS method, a subject has to report one of the three possible responses (increment, decrement, no change). Therefore, it is much more difficult for the subject to guess the response, and it is possible for the experimenter to check the response with the current direction of force (ascending or descending).

\subsection{Data Analysis}

The total number of scores in each interval is calculated by adding the number of hits, overshoots, and undershoots for all unnoticed trials. The average values of hits, overshoots, and undershoots are calculated, and normalized for all participants. The average percentage of overshoots (OS\%), undershoots (US\%), and their difference ((OS-US)\%) in the first interval (before force increment/decrement) and second interval (after force increment/decrement) are presented in Table 1. The effects of force increment/decrement on the OS\%, US\%, and (OS-US)\% are also presented for the two force directions (opposed and aid).

Based on the observations for the opposed force direction, the overshoots are decreased and increased by increasing and decreasing the sub-threshold forces on the subjects' hands, respectively. However, the undershoots are increased and decreased by increasing and decreasing the sub-threshold forces, respectively. It seems that a combination of the overshoots and undershoots can be a strong performance index to show the effects of sub-threshold forces on human actions. Therefore, the difference of overshoots and undershoots ((OS-US)\%) is calculated for each interval of the measurement period. As shown in Table 1, the (OSUS)\% is decreased/increased by increasing/decreasing the unnoticed forces for the opposed force direction. The overall performance index $(\Delta$ (OS-US)\% $)$ is the difference between the (OS-US)\% of the first interval (before force increment/decrement) and second interval (after force increment/decrement) of the measurement period in all unnoticed trials. The $\Delta$ (OS-US) $\%$ of all four combinations of the two factors in this study are calculated and presented in the last column of Table 1 .

The result is analyzed using the repeated-measures Analysis of Variance (ANOVA). A two-way repeated-measures ANOVA is conducted on the performance index to study the effects of sub-threshold forces on human performance. The analysis for all experiments is done at a significance level of 0.05. In the analysis of the data from the experiments, the log transform was used to correct for unequal variances between the groups and some slight non-normalities in the data. This log transform corrected all irregularities within the original data [14]. A sphericity test is conducted to check the sphericity assumption of the repeated-measures ANOVA. The chi-square approximation for this test is 8.81 and the associated p-value, 0.1167 , is greater than the alpha level 0.05. Thus, the data meet the sphericity assumption [3].

\section{RESULTS AND DISCUSSION}

In this study, a performance index $(\Delta(\mathrm{OS}-\mathrm{US}) \%)$ is defined to measure human performance during a Fitts' type task. The performance index results are presented in Table 1 for the force directions and force increment/decrement of sub-thresholds applied forces. It appears that the accuracy of subjects is affected when the applied forces on their hand increased or decreased. As shown in Table 1, the $\Delta$ (OS-US) $\%$ of the opposed force increment is $-3.51 \%$, and the $\Delta$ (OS-US) $\%$ of the opposed force decrement is $1.63 \%$. Based on the results, the $\Delta(\mathrm{OS}-\mathrm{US}) \%$ is increased when the applied force is decreased. The subjects obviously did less overshoots and more undershoots when the opposed force increased compared to the time that the opposed force decreased. In other words, the subjects' actions are affected when the opposed applied force on their hand increased.

As shown in Table 1, the $\Delta$ (OS-US) $\%$ of the aid force increment and decrement are $-1.74 \%$ and $-2.59 \%$, respectively. The results show that the $\Delta(\mathrm{OS}-\mathrm{US}) \%$ is decreased when the aid applied force is decreased. The subjects obviously did more undershoots and less overshoots when the aid force decreased compared to the time that the aid force increased. It is obvious that there is an interaction between the force direction and force increment/decrement factors. In other words, the effect of force direction on human performance is dependent on the increment or decrement of force. Results of the two-way repeated-measures ANOVA on $\Delta$ (OS$\mathrm{US}) \%, \mathrm{~F}(1,5)=28.33$ and $\mathrm{p}$-value $=0.0031$, also supports this trend. Consequently, four one-way repeated-measures ANOVAs are conducted on the $\Delta$ (OS-US) $\%$ to study the simple effects between each level of the force direction and the force increment/decrement.

The repeated-measures ANOVA on the increment or decrement of the opposed force, $\mathrm{F}(1,5)=15.83$ and $\mathrm{p}$-value = 0.0106 , show that there is definitely a difference between the effects of increasing and decreasing of the sub-threshold opposed forces on subjects' actions.

However, the results for the increment or decrement of the aid force, $\mathrm{F}(1,5)=3.39$ and $\mathrm{p}$-value $=0.1252$, show that there is no difference between the effects of increasing and decreasing of the sub-threshold aid forces on subjects' actions. As shown in Table 1, the difference of $\Delta$ (OS-US) $\%$ for the aid direction is very small. It is also shown in Ta- 
Table 1: The average of overshoots, undershoots, and their difference for force Direction and Increment/Decrement factors

\begin{tabular}{|c|c|c|c|c|c|c|c|c|c|c|c|}
\hline \multirow[t]{2}{*}{$\begin{array}{c}\text { Force } \\
\text { Dir }\end{array}$} & \multirow[t]{2}{*}{$\begin{array}{c}\text { Force } \\
\text { Inc/Dec }\end{array}$} & \multicolumn{3}{|c|}{$\begin{array}{c}\text { Before Force } \\
\text { Increment/Decrement }\end{array}$} & \multicolumn{3}{|c|}{$\begin{array}{c}\text { After Force } \\
\text { Increment/Decrement }\end{array}$} & \multicolumn{3}{|c|}{$\begin{array}{l}\text { Sub-threshold } \\
\text { Force Effects }\end{array}$} & \multirow[t]{2}{*}{$\Delta(\mathrm{OS}-\mathrm{US}) \%$} \\
\hline & & OS\% & US\% & (OS-US)\% & OS\% & US\% & $(\mathrm{OS}-\mathrm{US}) \%$ & $\mathrm{OS} \%$ & US\% & (OS-US)\% & \\
\hline opposed & Inc. & 5.43 & 1.25 & 4.18 & 3.23 & 2.56 & 0.67 & $\downarrow$ & $\uparrow$ & $\downarrow$ & -3.51 \\
\hline opposed & Dec. & 4.24 & 2.13 & 2.11 & 4.98 & 1.24 & 3.74 & $\uparrow$ & $\downarrow$ & $\uparrow$ & 1.63 \\
\hline Aid & Inc. & 4.37 & 1.28 & 3.10 & 3.9 & 2.55 & 1.36 & $\downarrow$ & $\uparrow$ & $\downarrow$ & -1.74 \\
\hline Aid & Dec. & 4.80 & 1.25 & 3.55 & 3.14 & 2.18 & 0.96 & $\downarrow$ & $\uparrow$ & $\downarrow$ & -2.59 \\
\hline
\end{tabular}

ble 1 that the increment of the aid force does not follow the same trend similar to other facotrs' levels. The overshoots increased, and undershoots decreased by increasing the aid force.

The one-way repeated-measures ANOVA results for the force direction of the force increment, $\mathrm{F}(1,5)=6.39$ and $\mathrm{p}$ value $=0.0527$, show that there is an approaching significant difference between the opposed and the aid forces in the case of force increment.

The one-way repeated-measures ANOVA results for the force direction of the force decrement, $\mathrm{F}(1,5)=22.11$ and $\mathrm{p}$-value $=0.0053$, indicates that there is statistically significant difference between the opposed and aid force directions, meaning that we can change the force direction to affect human performance.

\section{CONCLUSION}

The results of our study and analysis indicate that the human performance in haptic-enabled virtual reality applications can be affected using small sub-threshold controlled forces. In other words, we can pull/push the users' hand without them being aware of it into predictable positions by increasing/decreasing the sub-threshold opposed forces. For example, a control strategy can be employed to maintain a user's hand on a trajectory in a virtual environment using the opposed forces. The increment or decrement of opposed forces can be used to pull or push the user's hand on the trajectory. However, the force intensity must be tuned for a specific task.

Based on the results, there is an increase in errors even though the user is not aware of being manipulated. This study shows that the magnitude of the error may result from applying the sub-threshold forces. Therefore, there is a trade-off in applications. For example, in the case of friction compensation [15], too much or too little friction compensation can actually make things worse. In this case, overcompensation is shown from the results to be more problematic.

As mentioned in Section 5, there is an approaching significant difference between the opposed and aid forces in the case of force increment. Future work will investigate the results of this study with more subjects to see if there is any changes in the repeated-measures ANOVA results.

It appears from the results that the subjects do more overshoots and less undershoots when the aid force increased. Future work will study this trend in a full-scale study with more subjects. The improvement of performance indexes will also be investigated. In addition, the effects of base force intensity on human performance will also be studied.

\section{ACKNOWLEDGEMENTS}

The authors gratefully acknowledge the participation of subjects in the experiments.

\section{REFERENCES}

[1] Handshake VR Inc. . www.HandshakeVR.com.

[2] Sensable Technologies Inc. http://www.sensable.com.

[3] SAS/STAT User's Guide. Cary NC: SAS Institute Inc., fourth edition, 1989. Version 6.

[4] Allin S., Matsuoka Y., and Klatzky R. L. Measuring Just Noticeable Differences for Haptic Force Feedback: Implications for Rehabilitation. Symposium on Haptic Interfaces for Virtual Environment and Teleoperator Systems, pages 299-302, 2002.

[5] Bernstein R. S., and Gravel J. S. Method for determining hearing sensitivity in infants; The interweaving staircase procedure. Journal of the American Academy of Audiology, 1:138-145, 1990.

[6] Dachille F., Qin H., and Kaufman A. E. A novel haptics-based interface and sculpting system for physics-based geometric design. Computer-Aided Design, 33(5):403-420, 2001.

[7] Fitts P.M. The information capacity of the human motor system in controlling the amplitude of movement. Journal of Experimental Psychology, 47:381-391, 1954.

[8] Garbaya Samir and Zaldivar-Colado U. The affect of contact force sensations on user performance in virtual 
assembly tasks. Virtual Reality, 11(4):287-299, Oct. 2007.

[9] Gescheider G.A. Psychophysics: The Fundamentals. Lawrence Erlbaum Associates, 3rd edition, 1997.

[10] Ho Cristy, Tan Hong Z., Barbagli Federico, Salisbury Ken, and Spence Charles. Isotropy and visual modulation of haptic force direction discrimination on the human finger. Proceedings of the EuroHaptics Conference, pages 483-486, July 3-6 2006. Paris, France.

[11] Jandura L. and Srinivasan M. A. Experiments on Human Performance in Torque Discrimination and Control. Proc. of the ASME Dynamic Systems and Control Division, 55-1:369-375, 1994.

[12] Jones L. A. Matching forces: Constant errors and differential thresholds. Perception, 18(5):681-687, 1989.

[13] Kim J., Kim H., Tay B., Muniyandi M., Jordan J., Mortensen J., Oliveira M., Slater M., and Srinivasan M. Transatlantic Touch: A Study of Haptic Collaboration over Long Distance. Presence, 13(3), 2004. Special Issue: Collaborative Virtual Environments.

[14] Kuehl R. O. Design of experiments : statistical principles of research design and analysis. Pacific Grove, CA ; London : Duxbury/Thomson Learning, 2nd edition, 2000.

[15] Lawrence D. A., Pao L. Y., Dougherty A. M., Pavlou Y., Brown S. W., and Wallace S. A. Human Perceptual Thresholds of Friction in Haptic Interfaces. Proc. ASME Dynamic Systems and Control Division, 64:287-294, November 1998. ASME Int. Mech. Engr. Cong. and Expo., Anaheim, CA.

[16] Lederman S.J., Klatzky R.L., Hamilton C.L. and Ramsay G.I. Perceiving roughness via a rigid probe: Psychophysical effects of exploration speed and mode of touch. Haptics-e (Electronic Journal of Haptics Research), 1(1):1-20, 1999.

[17] Lee, Gregory S. and Hannaford, Blake. Preliminary two dimensional haptic thresholds and task performance enhancements. Haptics Symposium, March 2003.

[18] O'Toole R., Playter R., Krummel T., Blank W., Cornelius N., Roberts W., Bell W. and Raibert M. Assessing skill and learning in surgeons and medical students using force feedback surgical simulator. Medical Image Computing and Computer-Assisted Intervention (MICCAI), 1496:899-909, 1998. Springer.

[19] Pang X., Tan H.Z. and Durlach N. Manual discrimination of force using active finger motion. Perception 65 Psychophysics, 49(6):531-540, 1991.

[20] Paré M., Carnahan H., and Smith A. M. Magnitude estimation of tangential force applied to the fingerpad. Experimental Brain Research, 142:342-348, 2002.

[21] R.L. Williams II, M.-Y. Chen, and J.M. Seaton. Haptics-Augmented High School Physics Tutorials. Intl. Journal of Virtual Reality, 5(1), 2001.

[22] Salb T., Brief J.,Burgert O., Hassfeld S. and Dillmann R. Haptic based risk potential mediation for surgery simulation. Proceedings of: 1. International Workshop on Haptic Devices in Medical Applications (HDMA), June 1999. Paris, France.
[23] Sallnas E. Supporting Presence in Collaborative Environments by Haptic Force Feedback. $A C M$ Transactions on Computer-Human Interaction, 7(4):461-476, December 2000.

[24] Schiffman, Harvey Richard. Sensation and perception : an integrated approach. New York: Wiley, 5th edition, 2000.

[25] Schmidt, Richard A. and Lee, Timothy D. Motor control and learning : a behavioral emphasis. Champaign, IL; Windsor, ON : Human Kinetics, 4th edition, 2005.

[26] Srinivasan M. and Basgodan C. Haptics in Virtual Environments: Taxonomy, Research Status, and Challenges. Computers and Graphics, 21(4):393-404, 1997.

[27] Stanney K.M.,Mourant R.R.,and Kennedy R.S. Human factors issues in virtual environments: A review of the literature. Presence: Teleoper. Virtual Environ., 7(4):327-351, 1998.

[28] Wall S. and Harwin W. Quantification of the effects of haptic feedback during a motor skills task in a simulated environment. Proc. Second PHANToM Users Research Symposium, 2000.

[29] Wang D., Rossi M., Shu J., and Tuer K. Collaborative Tele-haptics - A Pilot Study Evaluation. VRIC- Laval Virtual 2005- 7th Virtual Reality International Conference, Laval, France, April 2005.

[30] Zadeh M.H., Wang D. and Kubica E. Human Factors for Designing a Haptic Interface for Interaction with a Virtual Environment. Haptic Audio Visual Environments and their Applications, 200\%. IEEE International Workshop on, 1:21-26, Oct. 2007.

[31] Zadeh M.H., Wang D. and Kubica E. Perception-Based Lossy Haptic Compression Considerations for Velocity-Based Interactions. Multimedia Systems Journal, 13(4):275-282, Jan. 2008. 